Journal of Russian Laser Research, Volume 18, Number 2, 1997

\title{
STATE OF AND PROSPECTS FOR XeF(C-A) OPTICALLY PUMPED LASERS
}

\author{
F. A. van Goor, ${ }^{1}$ S. V. Mitko, ${ }^{2}$ V. N. Ochkin, ${ }^{2}$ and W. J. Witteman ${ }^{1}$
}

${ }^{1}$ University of Twente, Department of Applied Physics, P.O. Box 217, 7500 AE Enschede, The Netherlands

${ }^{2}$ P. N. Lebedev Physical Institute, Russian Academy of Sciences, Leninsky Pr. 53, Moscow 117924, Russia

\begin{abstract}
Experimental and theoretical results are presented on an $\mathrm{XeF}(\mathrm{C}-\mathrm{A})$ blue-green laser driven by 5-kJ energy. The laser was pumped by a ferrite-induced discharge of $90 \mathrm{~cm}$ in length. The output energy of $0.22 \mathrm{~J}$ was obtained with a plane-parallel resonator. A program to simulate laser operation has been developed. Numerical results for a wide range of conditions are compared with experiments performed by us and by other authors. It is found that intracavity refractive losses limit laser operation for XeF pressures above 3 torr. The laser efficiency strongly depends on the discharge-to-cavity length ratio. Possible ways to increase the laser power and efficiency are discussed.
\end{abstract}

\section{Introduction}

An $\mathrm{XeF}(\mathrm{C}-\mathrm{A})$ optically pumped laser operating at $480 \mathrm{~nm}$ was first demonstrated in 1978 [1]. The fluorescence of $\mathrm{Xe}_{2}^{*}$ excited by an e-beam at $172 \mathrm{~nm}$ was used for photolysis of $\mathrm{XeF}_{2}$ and to produce $\mathrm{XeF}[\mathrm{B}(1 / 2)]$ and $\mathrm{XeF}[\mathrm{C}(3 / 2)]$ excited states. It was pointed out that this laser is potentially highly efficient, scalable, and tunable over a bandwidth greater than $40 \mathrm{~nm}$. Laser output energies of $\sim 1 \mathrm{~mJ}$ were obtained. Nearly simultaneously, broadband optical pumping based on an exploded wire technique was applied to get lasing with the $\mathrm{XeF}(\mathrm{C}-\mathrm{A})$ system. Energies of $4 \mathrm{~mJ}$ were obtained with a technical efficiency of $2 \cdot 10^{-7}$ [2]. Extensive experimental investigations of the exploded wire technique have resulted in energies up to $15 \mathrm{~J}$ with an efficiency of $4 \cdot 10^{-4}[3,4]$. The next step was taken in [5-7], where the laser was pumped by an open surface discharge capable of operating in a pulse repetitive mode. Energies of up to $117 \mathrm{~J}$ were obtained with an efficiency of $\sim 1.3 \cdot 10^{-3}$. Thus, an enlargement of the dimensions and input energies of experimental devices results in a significant increase of the laser efficiency.

Nowadays, the main trend in the development of the laser is diminution of sizes and increase of the pulse repetition frequency. Thus, a device $0.5-1 \mathrm{~m}$ in length with an input energy of 1-5 $\mathrm{kJ}$ has become an object of experimental investigations. The first experiments with a compact laser demonstrated an efficiency of about $5 \cdot 10^{-5}[8,9]$. Up to now, the reason for the low efficiency is not clear.

Despite numerous experimental works, theoretical analysis of this laser was begun just a few years ago. Actually, there is only one theoretical study that considers the pumping source and laser radiation parameters in addition to laser operation cycle [10]. Calculations for two experimental situations are made in this paper. The intracavity losses were introduced as a fitting parameter to get an agreement between the calculations and experiments. A value of these losses was chosen for each particular calculation. An analytical theory given in [4] considers lasing as a steady-state process and neglects gain spatial variations. Thus, application of the results $[4,10]$ to a wide range of experimental conditions is problematic. In order to avoid this drawback, we have developed a numerical code simulating the laser operation and verified it for various experimental conditions. In this paper, we consider a $\mathrm{XeF}(\mathrm{C}-\mathrm{A})$ laser with a plane-parallel resonator pumped by open single-channel surface discharge.

Translated from a manuscript submitted May 25, 1996. 


\section{The Pumping Source}

Experiments show that $\mathrm{XeF}_{2}$ molecules dissociate under the action of discharge radiation in the form of a bleaching wave that proceeds as an almost perfect hollow semicylinder [7]. This means that the pumping discharge is not cptically thick and cannot be considered as a black body radiator. Actually, if we assume, according to [10], a black body radiator, then the speed of the bleaching wave in the direction normal to the surface would be twice as high as that in a tangential direction. Hence, the wave front in this case is not a perfect semicylinder. The speed of the bleaching wave expansion is much higher than that of the discharge. For typical experimental conditions, it equals $\sim 10 \mathrm{~km} / \mathrm{s}$, whereas the discharge speed is approximately $1 \mathrm{~km} / \mathrm{s}$ [3-7].

Thus, the pumping discharge can be considered as an infinitely thin linear light source. This source is characterized by the number of photons emitted per unit body angle and unit spectral region for a unit time interval from a unit length $W(t)$ [photon $/ \mathrm{s} \cdot \mathrm{cm} \cdot \mathrm{ster} \cdot \mathrm{nm}]$. In this case, the equation for the $\mathrm{XeF}_{2}$ photodissociation has the form

$$
\frac{d N}{d t}=-\frac{2 N}{R} \int_{0}^{\pi / 2} d \theta \int_{0}^{\infty} d \lambda W(t, \lambda, \theta) \sigma(\lambda) \exp \left\{-\frac{\sigma(\lambda)}{\cos \theta} \int_{0}^{R} d r N(R, t)\right\},
$$

where $N$ is the density of the $\mathrm{XeF}_{2}$ molecules, $\sigma(\lambda)$ is the spectrum-dependent absorption cross-section, $R$ is the radius, and $W(t, \lambda, \theta)$ is the discharge intensity, which is dependent on time $t$, wavelength $\lambda$, and angle $\theta$. The condition $\theta=0$ corresponds to a normal direction with respect to the discharge axis. Assuming that $W$ does not vary significantly over the $\mathrm{XeF}_{2}$ absorption band of $130-180 \mathrm{~nm}$ and is constant within the angle interval $0-\pi / 2$, Eq. (1) can be rewritten as follows:

$$
\begin{aligned}
\frac{d n}{d \tau}=-\frac{n}{r} F, \quad F & =\frac{2}{\pi \Delta \lambda} \int_{0}^{\pi / 2} d \theta \int_{0}^{\infty} d \lambda \Sigma(\lambda) \exp \left\{-\frac{\Sigma(\lambda)}{\cos \theta} \int_{0}^{r} d r n\right\}, \\
r & =R N_{0} \sigma_{\max }, \quad \tau(t)=\pi N_{0} \sigma_{\max }^{2} \Delta \lambda \int_{0}^{t} d t W(t),
\end{aligned}
$$

where new dimensionless variables are introduced: $n=N / N_{0}, N_{0}$ is initial $\mathrm{XeF}_{2}$ density, $\Sigma(\lambda)=\sigma(\lambda) / \sigma_{\max }$, $\sigma_{\max }$ is the maximum absorption cross-section, and $\Delta \lambda=\int d \lambda \Sigma(\lambda)$ is the absorption bandwidth. In our calculations, we used the following approximation of experimental data taken from [3]:

$$
\Sigma(\lambda)=\frac{\exp \left[-\pi\left(\lambda-\lambda_{0}\right)^{2}\right]}{(\Delta \lambda)^{2}}, \quad \sigma_{\max }=6.7 \cdot 10^{-17} \mathrm{~cm}^{2}, \quad \text { and } \quad \Delta \lambda=19.46 \mathrm{~nm} .
$$

Therefore, in view of (2), we must find the temporal and spatial distributions of the absorbing molecules only once. All other cases can be obtained by substituting $N_{0}$ and $W(t)$ into (3).

Let us define the dimensionless radius $r$ of the bleaching wave as the radius that corresponds to the maximum pumping rate $d n / d \tau$. A dependence of $r$ on time $\tau$ is shown in Fig. 1. Certain specific regions of $r$ variations are presented in this plot. If $\tau$ is less than unity, then $r=\tau$. The formula $r=\tau^{0.5612}$ presents a good fit to the calculated data for $\tau$ ranging from 10 to 1000. As $\tau$ becomes larger than $1500, r(\tau)$ converges to $r=1.518 \cdot \tau^{0.5}$.

The function $r(\tau)$ is useful in determining the intensity of the pumping source $W(t)$ from the experimentally measured radius $R(t)$ and speed $d R / d t$ of the bleaching wave. In the particular case of $r=a \tau^{k}$, where $a$ and $k$ are constants, $W(t)$ can be expressed in terms of the measured parameters as follows:

$$
W(t)=\frac{d R}{d t} \frac{\left(R N_{0}\right)^{(1-k) / k}}{k \pi \Delta \lambda}\left(\sigma_{\max }\right)^{(1-2 k) / k} \frac{1}{a^{1 / k}}
$$




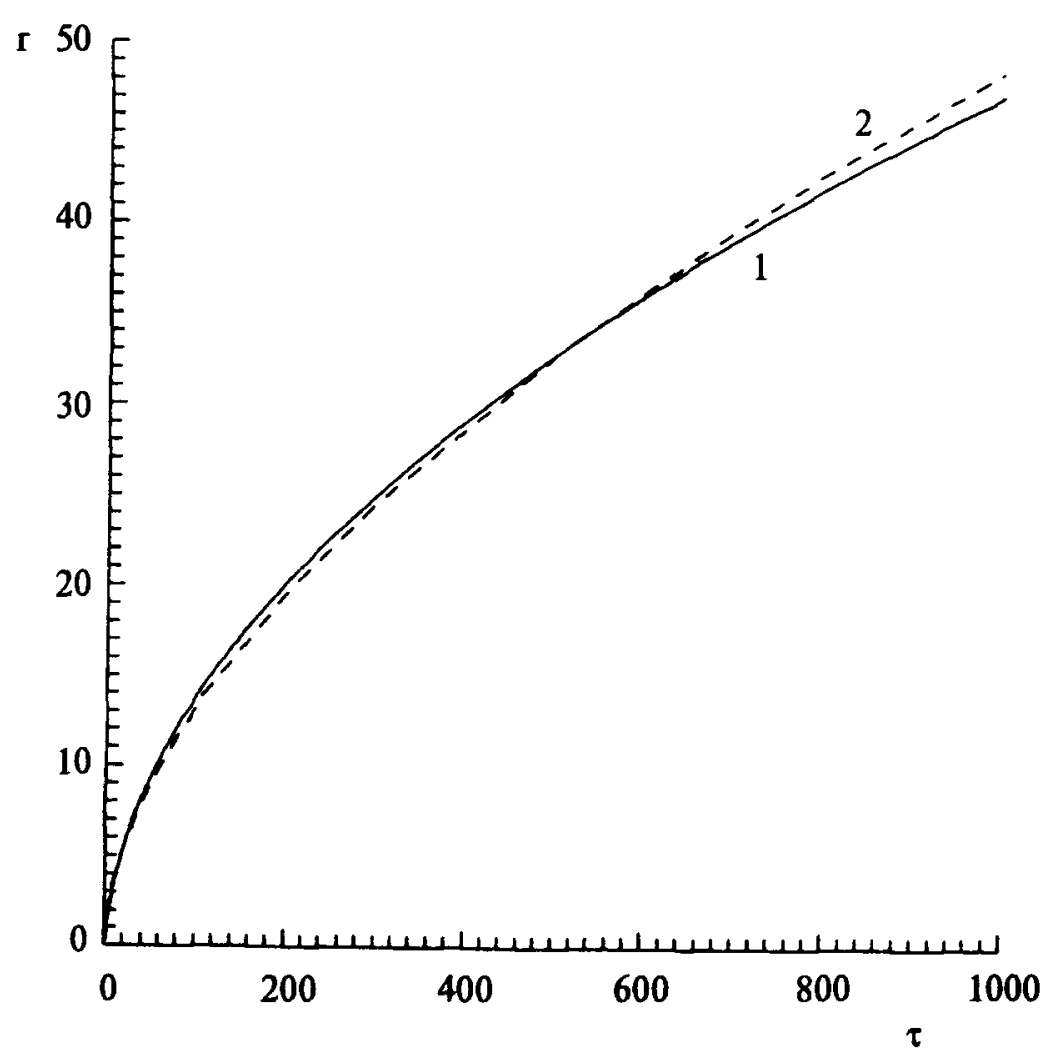

Fig. 1. The radius of the bleaching wave $r$ versus time $\tau: 1$ ) calculation and 2) fitting curve $r=\tau^{0.5612}$. Dimensionless scale.

For $k=0.5$, formula (4) presents a relation similar to that obtained analytically in [4] assuming a stationary profile of the bleaching wave:

$$
W(t)=\frac{2}{\pi \Delta \lambda} R \frac{d R}{d t} N_{0} \frac{1}{a^{2}} .
$$

It should be noted, that Eq. (5) is valid in a limited region. For conventional experimental parameters (see below) $W \sim 10^{21}-10^{23}$ photon $/ \mathrm{s} \cdot \mathrm{cm} \cdot \mathrm{nm} \cdot \mathrm{ster}, N_{0} \sim 10^{16}-10^{17} \mathrm{~cm}^{-3}$, and a pumping-pulse duration of $\sim 2 \mu \mathrm{s}, \tau$ changes from 6 to 5000 and the coefficients $a$ and $k$ are not constant. Therefore, the use of simplified relation (5) in processing experimental data leads to the false conclusion that $W(t)$ depends on the initial concentration $N_{0}$.

\section{Elementary Processes in $\mathrm{XeF}(\mathrm{C}-\mathrm{A})$ Laser}

Principal reactions and rate constants that are of importance in the kinetics of $\mathrm{XeF}(\mathrm{C}-\mathrm{A})$ optically pumped lasers were taken from [10]. A few modifications were made compared to that paper:

One absorption band of $\mathrm{XeF}_{2}$ centered at $158 \mathrm{~nm}$ was taken into account, because the absorption of photons at $230 \mathrm{~nm}$ does not produce excited states of $\mathrm{XeF}(\mathrm{B}, \mathrm{C}, \mathrm{D})$ [11].

It was shown in [10] that the working mixture temperature in the course of $\mathrm{XeF}_{2}$ photodissociation does not exceed $100 \mathrm{~K}$; hence, all temperature-dependent parameters are replaced by their corresponding values at room temperature.

Quenching of $\mathrm{XeF}(\mathrm{B}, \mathrm{C})$ by binary collisions with $\mathrm{Ar}$ and $\mathrm{N}_{2}$ was included in the kinetic model. Reactions and rate constants used in our model are listed in Appendix along with kinetic equations for particle 
concentrations.

\section{Laser Flux Dynamics}

An equation describing laser intracavity flux has the following form [13]:

$$
\frac{d \Phi}{d t}=c \frac{L_{a}}{L_{c}}\left(g+\frac{1}{2 L_{a}} \ln \left(R_{1} R_{2}\right)\right) \Phi-c \alpha \Phi+c \frac{L_{a}}{L_{c}} g \frac{1}{V_{m}},
$$

where $\Phi$ is the density of photons averaged over the laser cavity length, $c$ is the speed of light, $R_{1}$ and $R_{2}$ are the mirror reflection coefficients, $g=\sigma_{\mathrm{se}}[\mathrm{XeF}(\mathrm{C})]$ is gain coefficient, $\sigma_{\mathrm{se}}$ is the stimulated emission crosssection at the center of the laser line, $[\mathrm{XeF}(\mathrm{C})]$ is the population of the $\mathrm{C}$-state, $L_{c}$ and $L_{a}$ are the cavity and active medium lengths, respectively, and $\alpha$ is the intracavity loss coefficient (see Sec. 6). The last term in (6) represents the contribution of spontaneous emission, $V_{m}=L_{c} S$ is the volume of lasing region, where $S$ is the cross-section of the bleaching wave covering a clear laser aperture. The density of laser output power $P_{\text {out }}$ is given by the expression

$$
P_{\text {out }}=\frac{\operatorname{ch} \nu}{2} \Phi \ln \left(\frac{1}{R_{1} R_{2}}\right)
$$

The optical flux build-up time of such a laser can be estimated by the relation [12]

$$
T \simeq \frac{G L_{c}}{c g L_{a}}
$$

where $T$ is the build-up time and $G$ is laser emission-to-spontaneous emission ratio. It has been shown [12] that $G \sim 18 \pm 1$ for lasers with $L_{c} \sim 0.5-2 \mathrm{~m}$ in the visible spectral region. The gain of the C-A transition obtained in experiments is low $\left(\sim 0.003 \mathrm{~cm}^{-1}\right)$ due to the small gain cross-section $\left(\sim 10^{-17} \mathrm{~cm}^{2}\right)$ and broad gain spectral width $(\sim 70 \mathrm{~nm})[5]$. By substituting $g=3 \cdot 10^{-3} \mathrm{~cm}^{-1}, G=18$, and $L_{c} / L_{a}=2$ into Eq. (6), we obtain $T=0.4 \mu \mathrm{s}$. Hence, the build-up time is comparable to the pumping pulse duration $\sim 1 \mu \mathrm{s}$, which limits the laser efficiency. Thus, a correct theoretical analysis of $\mathrm{XeF}(\mathrm{C}-\mathrm{A})$ laser operation should take into consideration the photon flux dynamics. It is seen from Eq. (8) that the build-up time depends on $L_{a} / L_{c}$. Therefore, laser output power also depends on this ratio. These factors have not been taken into account in previous papers.

\section{Active Medium Inhomogeneity}

It has been found that small refractive index gradients $\sim 10^{-7} \mathrm{~cm}^{-1}$ arise in the active medium of a XeF laser in the course of $\mathrm{XeF}_{2}$ dissociation $[4,7,14]$. Convincing experiments with plane-parallel and plane-conic resonators have proved that these gradients are principally responsible for internal losses, so-called refractive losses $[4,15]$. There are two main reasons for refractive index variations in a bleaching wave in $\mathrm{XeF}_{2}$. The first one is associated with different polarizabilities of the parent $\mathrm{XeF}_{2}$ molecule and the combined polarizability of the photodissosiation products. The chemical composition changes from $\mathrm{XeF}_{2}$ molecule to xenon atom

and two fluorine atoms during bleaching-wave-front passage. Thus, the chemical component $\Delta n_{\mathrm{ch}}$ of the refractive index change is as follows:

$$
\Delta n_{\mathrm{ch}}=\beta_{\mathrm{Xe}}[\mathrm{Xe}]+2 \beta_{\mathrm{F}}[\mathrm{F}]-\beta_{\mathrm{XeF}_{2}}\left[\mathrm{XeF}_{2}\right]
$$


where $\beta_{\mathrm{Xe}}, \beta_{\mathrm{F}}$, and $\beta_{\mathrm{XeF}_{2}}$ are the polarizabilities of the particles, [Xe] and [F] are final concentrations of the products, and $\left[\mathrm{XeF}_{2}\right]$ is the initial concentration of $\mathrm{XeF}_{2}$. The conservation of atoms yields:

$$
\Delta n_{\mathrm{ch}}=\left(\beta_{\mathrm{Xe}}+2 \beta_{\mathrm{F}}-\beta_{\mathrm{XeF}_{2}}\right)\left[\mathrm{XeF}_{2}\right]
$$

Thus, the variation in the refractive index caused by changed chemical composition is proportional to the initial $\mathrm{XeF}_{2}$ concentration. The absolute value of this variation is approximately $1.5 \cdot 10^{-7}$ at an initial pressure of 2 torr of $\mathrm{XeF}_{2}$ [4]. One more reason is the increase in gas mixture pressure caused by heat release in the process of $\mathrm{XeF}_{2}$ photodissociation. The increased pressure results in gas density perturbations and a corresponding change in the refractive index $\Delta n_{g}$. We will employ the following expression for estimating $\Delta n_{g}[15]:$

$$
\Delta n_{g}=\left(n_{0}-1\right) \frac{Q(\gamma-1)}{\rho D^{2}},
$$

where $n_{0}$ is the initial refractive index of the mixture, $D$ is the bleaching wave speed, $\rho$ is the initial density of the mixture, $\gamma$ is the adiabatic exponent, and $Q$ is the heat release per unit volume. For the widely used buffer gas mixture $\left[\mathrm{N}_{2}\right]:[\mathrm{Ar}]=1: 3, \gamma-1 \simeq 0.57$ and $\left(n_{0}-1\right) / \rho \simeq 0.17 \mathrm{~cm}^{3} / \mathrm{g}$ [16]. The upper limit for the heat release is $Q \sim\left[\mathrm{XeF}_{2}\right] h \nu$, where $h \nu$ is the energy of a photon at a wavelength of $158 \mathrm{~nm}$. At the initial concentration of $\mathrm{XeF}_{2}$ molecules at 2 torr, $Q \simeq 8 \cdot 10^{-2} \mathrm{~J} / \mathrm{cm}^{3}$. In the conditions of our experiments (see Sec. 7.1), the bleaching wave speed is approximately $D \simeq 14 \mathrm{~km} / \mathrm{s}$. In this case, the gas-dynamic contribution to refractive index change is $\Delta n_{g} \sim 4 \cdot 10^{-8}$. It is seen that in the conditions of our experiments the gas dynamic contribution to refractive index variation is negligible as compared to that due to the breaking of chemical bonds in $\mathrm{XeF}_{2}$. Therefore, we will assume a linear dependence of $\Delta n$ on the initial $\mathrm{XeF}_{2}$ concentration. In general, the problem concerning optical inhomogeneities requires further investigation.

\section{Intracavity Losses}

Refractive index variation leads to intracavity losses. The refractive losses are described by the formula [17]

$$
\alpha=\frac{1}{w}\left(2 \Delta n \frac{L_{a}}{L_{c}}\right)^{1 / 2}
$$

where $w$ is the width of the lasing region. In our numerical model, $w$ is defined as the half-height width of the spatial distribution of $\mathrm{dN} / \mathrm{dt}$ (see Sec. 2). Because of the lack of data on polarizabilities $\beta_{\mathrm{Xe}}, \beta_{\mathrm{F}}$, and $\beta_{\mathrm{XeF}_{2}}$ we used the following approximation for $\Delta n$ :

$$
\Delta n=\varepsilon\left[\mathrm{XeF}_{2}\right],
$$

where $\left[\mathrm{XeF}_{2}\right]$ is the initial $\mathrm{XeF}_{2}$ concentration and $\varepsilon=2.9 \cdot 10^{-7} \mathrm{~cm}^{3}$ is the coefficient that provides the best agreement between the calculations and experimental results.

\section{Experimental Results. Comparison with Calculation}

\section{1. $\mathrm{XeF}(\mathrm{C}-\mathbf{A})$ Laser with 5-kJ Input Energy}

A detailed description of the installation and experimental technique is given in [9]. The laser was powered by $5-\mathrm{kJ}$ energy stored in a capacitor bank. A pumping discharge was initiated by a $90 \cdot \mathrm{cm}$-long ferrite rod. The rod was positioned at the edge of the clear laser aperture, which was $8.5 \mathrm{~cm}$ in diameter. 


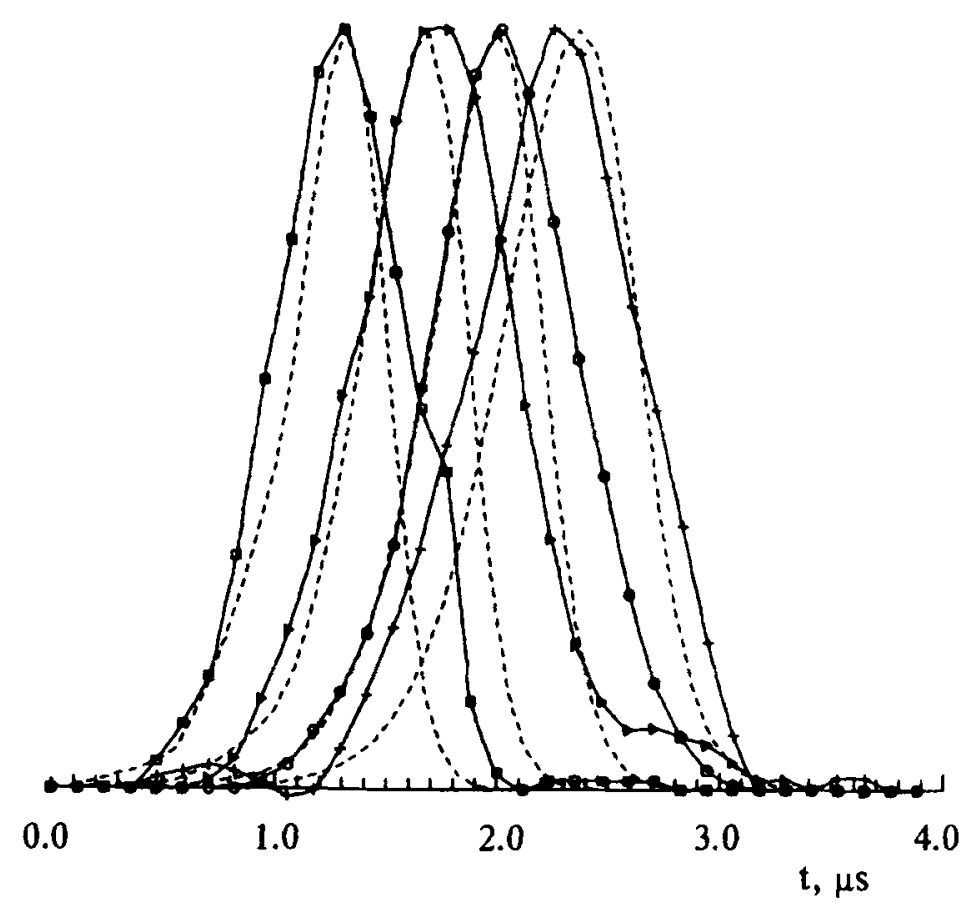

Fig. 2. C-A luminescence at different distances from the ferrite surface versus time: $8.6 \mathrm{~mm}(\square), 13.6 \mathrm{~mm}(\triangleright), 18.6 \mathrm{~mm}$ $(o)$, and $24.3 \mathrm{~mm}(+)$. Dashed curves represent the results of calculations.

Because pumping source parameters are important factors affecting laser performance, we measured the pumping pulse $W(t)$ in our experimental conditions. Since determination of the pumping power from the measured speed and radius of the bleaching wave is not accurate (see Sec. 1), we used a modified approach. The pumping pulse shape $I(t)$ was measured at a wavelength of $156 \mathrm{~nm}$ in a mixture $\mathrm{N}_{2}: \mathrm{Ar}=1: 3$ at a total pressure $0.65 \mathrm{~atm}$. Then, the shapes of the $\mathrm{C}-\mathrm{A}$ luminescence pulses were measured at various distances from the discharge at 2.1-torr pressure of $\mathrm{XeF}_{2}$ added to the mixture. The shapes of $\mathrm{C}$-A luminescence are plotted in Fig. 2 by solid curves. The absolute value of the pumping flux $W(t)$ was obtained by choosing the maximum value of the experimentally measured intensity $I(t)$ that provided the best agreement between the observed and calculated luminescence pulses. The calculated pulse shapes of $\mathrm{C}-\mathrm{A}$ luminescence are shown in Fig. 2 by dashed curves at peak value $W(t)=5.5 \cdot 10^{21}$ photon $/ \mathrm{s} \cdot \mathrm{cm} \cdot \mathrm{nm} \cdot \mathrm{ster}(6.91 \mathrm{~kW} / \mathrm{cm} \cdot \mathrm{nm} \cdot \mathrm{ster})$.

The resulting intensity $W(t)$ of the pumping discharge is shown in Fig. 3 (curve 1). The corresponding calculated trace of the bleaching wave is shown in Fig. 4. In Figs. 5 and 6, the calculated profiles of smallsignal gain for this laser are also shown. It is seen that the gain reaches $\sim 1 \% \mathrm{~cm}^{-1}$ at a separation $\sim 1 \mathrm{~cm}$ from the ferrite surface and exists for $\sim 1 \mu \mathrm{s}$. The calculated energy of laser generation was then compared with experimental data for laser operation in the same mixture. In these experiments, the plane-parallel resonator consisted of two dielectric mirrors separated by a distance of $174 \mathrm{~cm}$. At the $\mathrm{XeF}(\mathrm{C}-\mathrm{A})$ emission wavelength, one mirror had $99.9 \%$ reflection and the other output mirror had a transmittance of $5 \%$. The transmittance of both mirrors at the $350-\mathrm{nm}$ wavelength of the competing $\mathrm{XeF}(\mathrm{B}-\mathrm{X})$ transition was higher than $85 \%$. Because the experimental conditions and the pumping source intensity were well defined, this comparison was used to determine the coefficient $\varepsilon$ of proportionality between the refractive index variation and the initial density of $\mathrm{XeF}_{2}$ in relation (13). The experimentally measured laser energy was $225 \mathrm{~mJ}$, while the calculation performed at $\varepsilon=2.9 \cdot 10^{-7} \mathrm{~cm}^{3}$ yielded $220 \mathrm{~mJ}$.

To verify our method in a wider range of experimental conditions, we simulated the operation of a more 


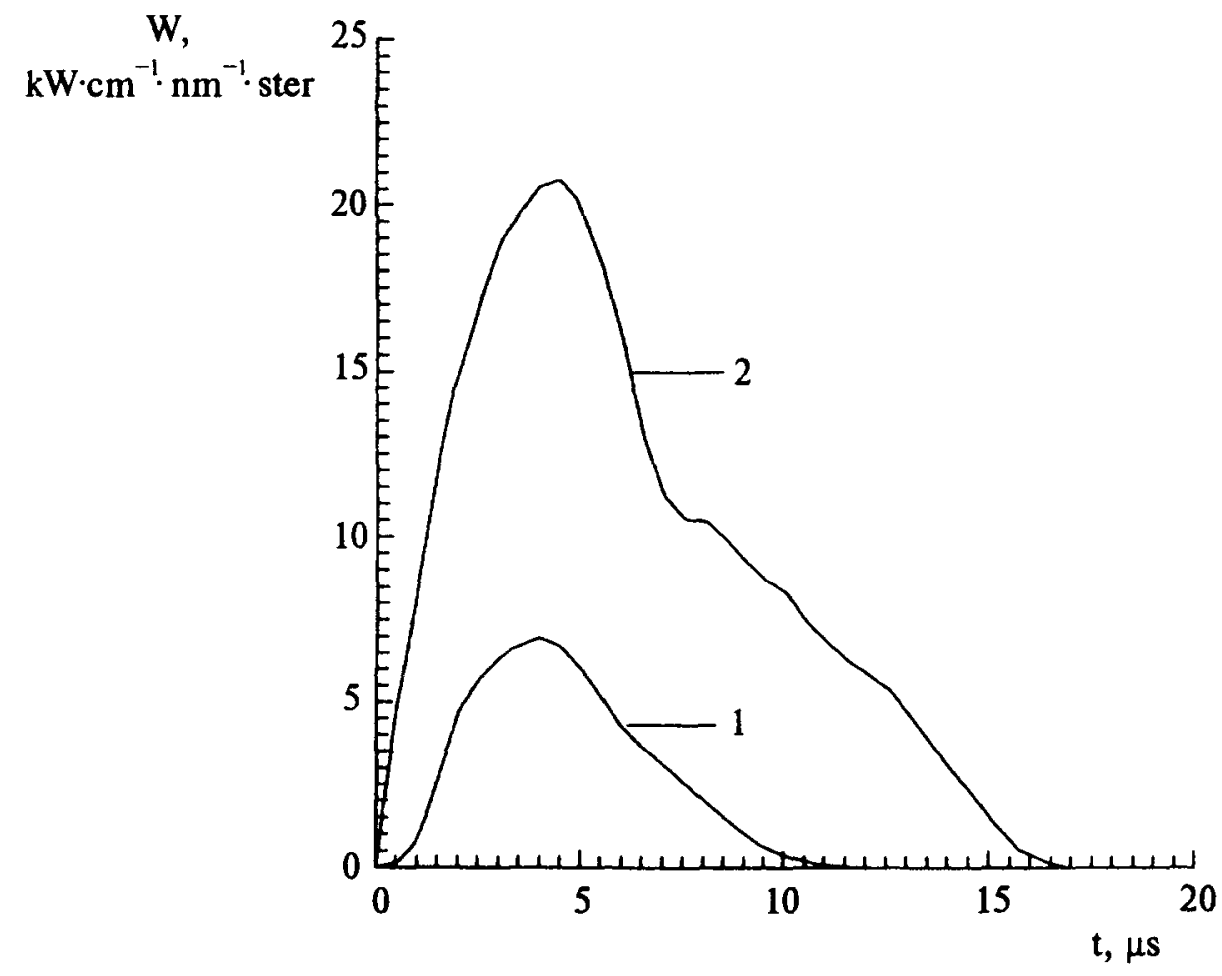

Fig. 3. The intensity of the pumping pulse $W$ versus time: 1) energy input of $5 \mathrm{~kJ}$ and 2) of $90 \mathrm{~kJ}$ [7].

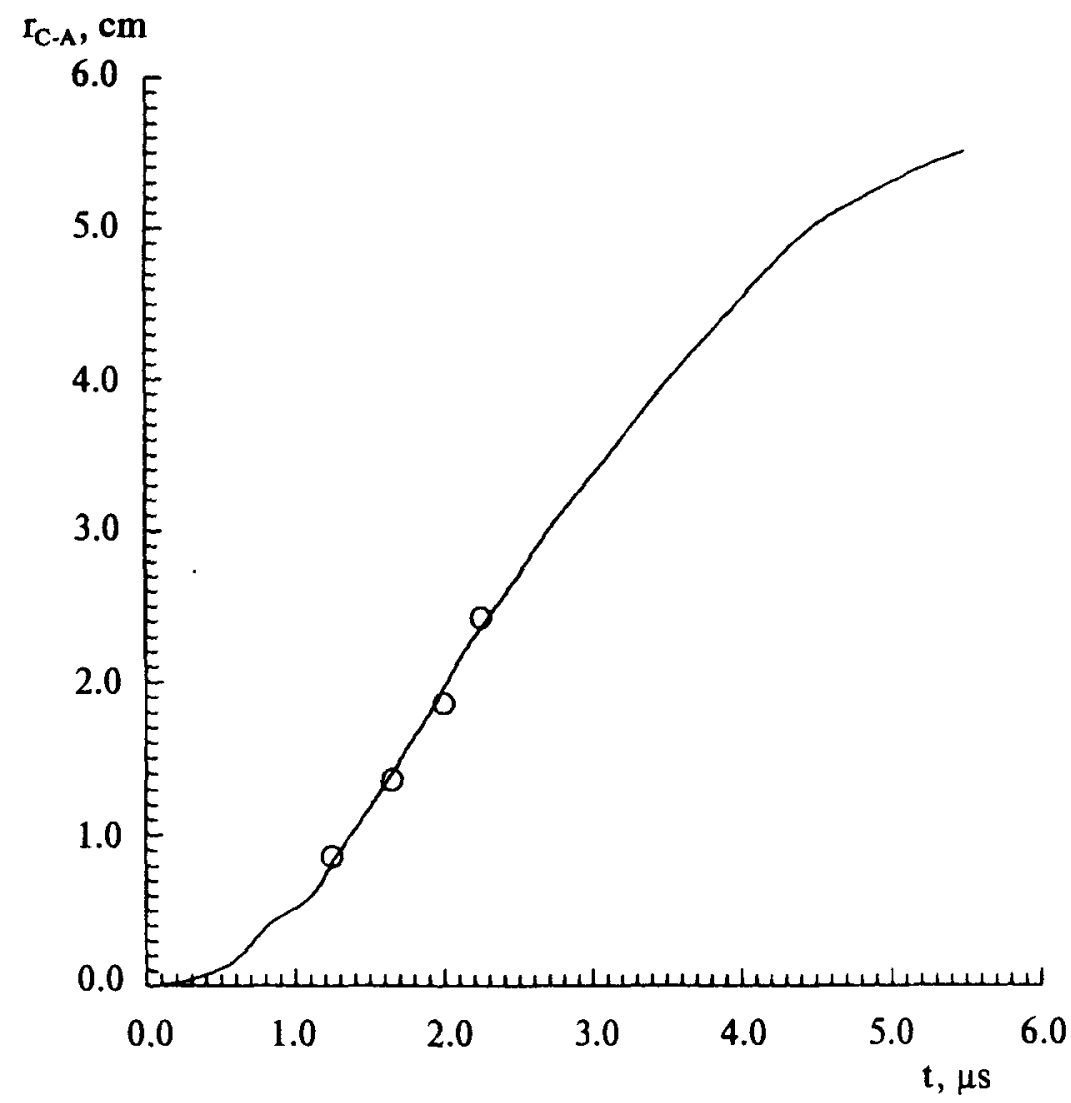

Fig. 4. Radius of $\mathrm{C}-\mathrm{A}$ luminescence wave versus time, calculation (solid curve) and measurement (o) data. $\left[\mathrm{XeF}_{2}\right]=$ 2.1 torr. 


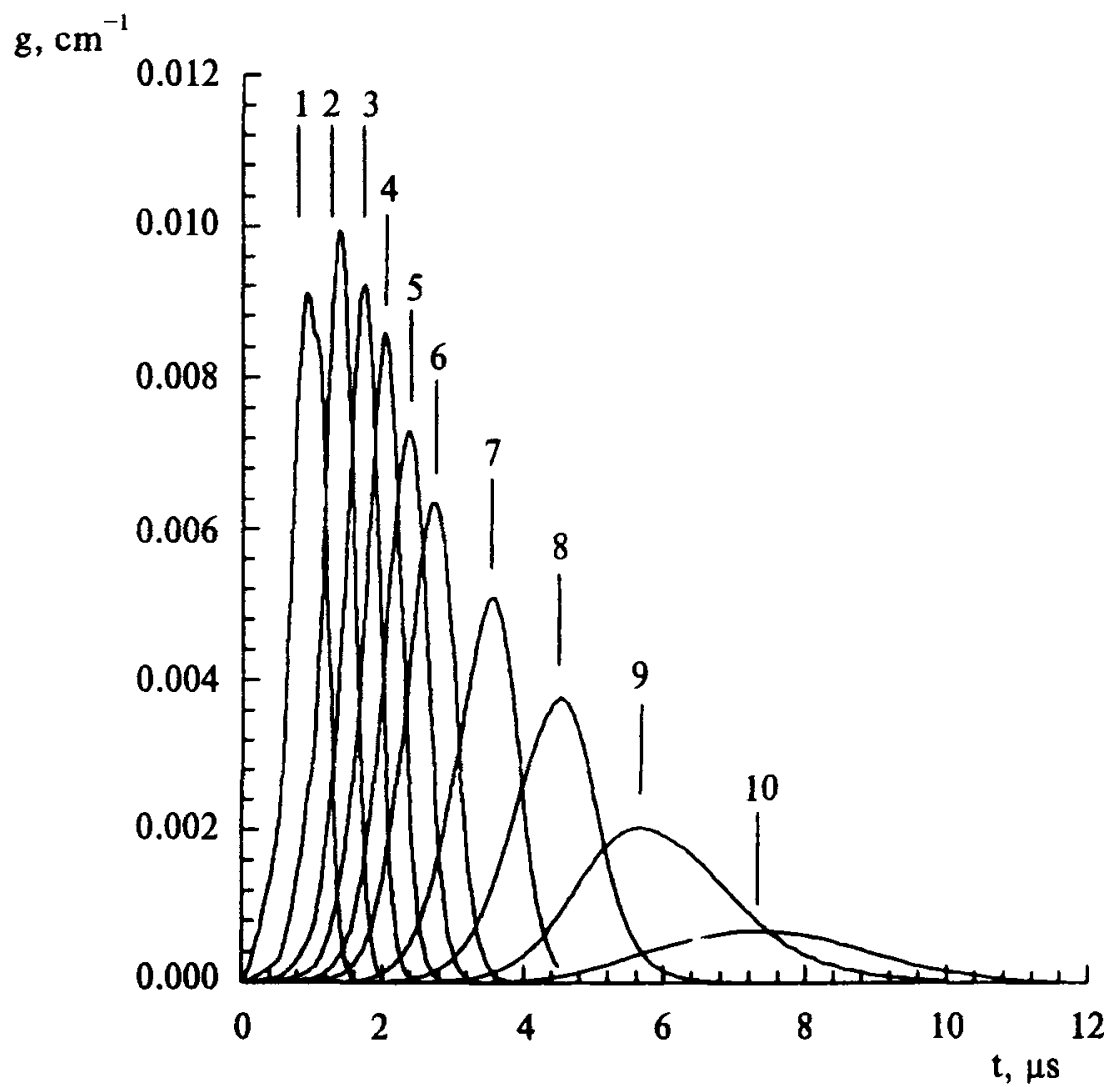

Fig. 5. Small signal gain on C-A transition versus time at different distances from the ferrite surface: 1 ) $0.5 \mathrm{~cm}$, 2) $1.0 \mathrm{~cm}, 3) 1.5 \mathrm{~cm}, 4) 2.0 \mathrm{~cm}, 5) 2.5 \mathrm{~cm}, 6) 3.0 \mathrm{~cm}, 7) 4.0 \mathrm{~cm}, 8) 5.0 \mathrm{~cm}, 9) 6.0 \mathrm{~cm}$, and 10) $7.0 \mathrm{~cm}$.

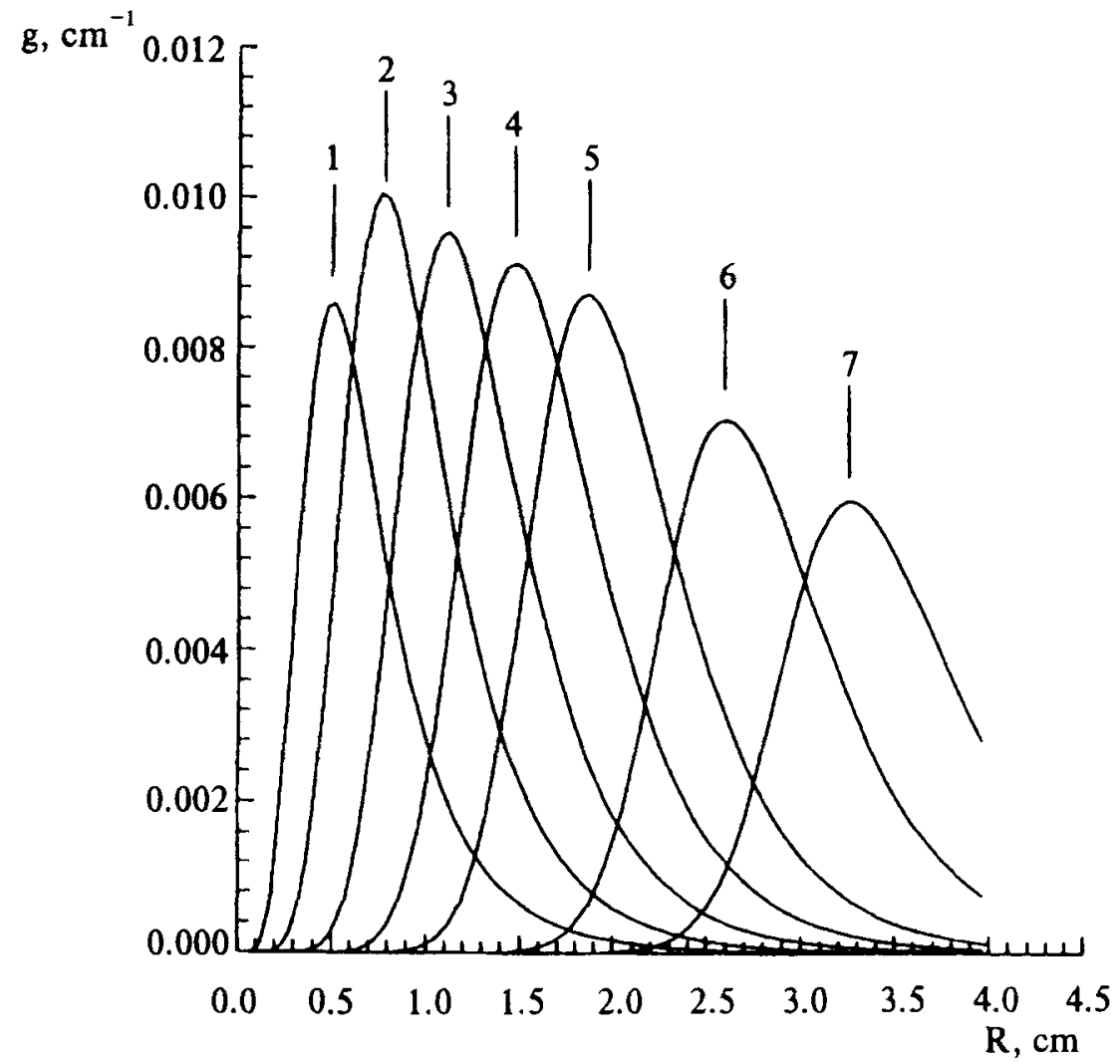

Fig. 6. Small signal gain on $\mathrm{C}-\mathrm{A}$ transition versus distance from the ferrite surface at different times: 1 ) $1.0 \mu \mathrm{s}$, 2) $1.25 \mu \mathrm{s}, 3) 1.5 \mu \mathrm{s}, 4) 1.75 \mu \mathrm{s}, 5) 2.0 \mu \mathrm{s}, 6) 2.5 \mu \mathrm{s}$, and 7) $3.0 \mu \mathrm{s}$. 
powerful laser.

\section{2. $\mathrm{XeF}(\mathrm{C}-\mathrm{A})$ Laser with $\mathbf{9 0 - \mathrm { kJ }}$ Input Energy}

Since experiments with small-scale lasers were used to determine the coefficient $\varepsilon$ in (13), which is the only fitting parameter in the numerical code, we had to compare our computation results with the experimental data of other authors. It was desirable that their experimental conditions be different from ours.

The most detailed description of experiments with large-scale lasers is given in [6, 7]. The laser was pumped by a $190-\mathrm{cm}$ long surface creeping discharge. The built-in optical resonator was $280 \mathrm{~cm}$ long and had two plane dielectric mirrors with 0.99 and 0.77 reflection and an optical diameter $20 \mathrm{~cm}$. The laser was filled with a mixture $\mathrm{N}_{2}: \mathrm{Ar}=1: 3$ at a total pressure of $1.4 \mathrm{~atm}$ and with variable contents of $\mathrm{XeF}_{2}$. The pumping source was placed on a side wall of the working chamber $(12 \times 20 \mathrm{~cm})$ at a distance of $6 \mathrm{~cm}$ from the resonator optical axis. The discharge was powered by an energy of $90 \mathrm{~kJ}$. To determine the pumping source parameters, we used the pumping pulse shape reported in [7] taking into account that the efficiency of an open discharge exhibits a rather weak dependence on its length, the electrical input, and the pressure of ambient gas. According to [7], this efficiency is $10 \%$ in the pumping band of $\mathrm{XeF}_{2}$. Thus, we calculated the total quantity of dissociated $\mathrm{XeF}_{2}$ molecules that corresponded to $10 \%$ efficiency. Then the peak intensity of the experimental dependence $W(t)$ was fitted in order to obtain this quantity of dissociated molecules. This yielded the peak value $W(t)=1.65 \cdot 10^{22}$ photon $/ \mathrm{s} \cdot \mathrm{cm} \cdot \mathrm{nm} \cdot \mathrm{ster}(20.7 \mathrm{~kW} / \mathrm{cm} \cdot \mathrm{nm} \cdot \mathrm{ster})$. The pumping pulse intensity is shown in Fig. 2 (curve 2).

The dependence of the output laser energy on the $\mathrm{XeF}_{2}$ initial pressure is shown in Fig. 7. The points represent experimental results from [7], and solid curve 1 is the result of our calculation. Calculations were performed at the same value of the coefficient $\varepsilon$ from Eq. (13) as in Sec. 7.1. In view of the fact that the accuracy of the experiments is approximately $20 \%$ and the accuracy of known reaction rates is $20-30 \%$, the agreement between our calculations and experimental data may be considered satisfactory. In order to illustrate the behavior of the output energy, the spatial profiles of the output energy density at different $\mathrm{XeF}_{2}$ pressures are shown in Fig. 8. Thus, the decrease in the output energy at high $\mathrm{XeF}_{2}$ pressure is explained by growing refractive losses, whereas the energy drop at low pressure is due to the small size of the laser chamber used in the experiment. Because of the low $\mathrm{XeF}_{2}$ pressure, only a small number of pumping photons are absorbed inside the laser chamber. Without this limitation, the calculations yield a substantially higher output energy of the laser described in [7]. The result of these calculations is shown in Fig. 7 by dashed curve 2 .

\section{Prospects for Higher Laser Efficiency}

Calculations performed for a laser driven by $5-\mathrm{kJ}$ energy show that an output energy of $1.3 \mathrm{~J}$ can be obtained at a $\mathrm{XeF}_{2}$ pressure of 1 torr. If the limiting effect of the laser aperture is neglected, an energy of $2 \mathrm{~J}$ can be obtained. Thus, further enlargement of laser dimensions is desirable and the development of a compact device seems to be impossible.

On the other hand, there is a way to increase laser efficiency by reducing the build-up time of the intracavity photon flux (see Sec. 4). To verify this approach, we calculated the output energy for the case where the cavity length $L_{c}$ is equal to the discharge length $L_{a}$. The calculation results for a 90-kJ-driven laser with $L_{a}=L_{c}=190 \mathrm{~cm}$ are shown in Fig. 7 . Solid curve 3 corresponds to the laser aperture reported in [7] and the case of an increased aperture corresponds to dashed curve 4. A considerable increase in output energy is obtained due to reduction of the laser dimensions.

Similar calculations performed for a laser driven by 5 -kJ energy yield even more promising results. At $L_{a}=L_{c}=90 \mathrm{~cm}$, an output energy of $6 \mathrm{~J}$ can be extracted with a laser aperture of $8.5 \mathrm{~cm}$ and that of $12 \mathrm{~J}$ 


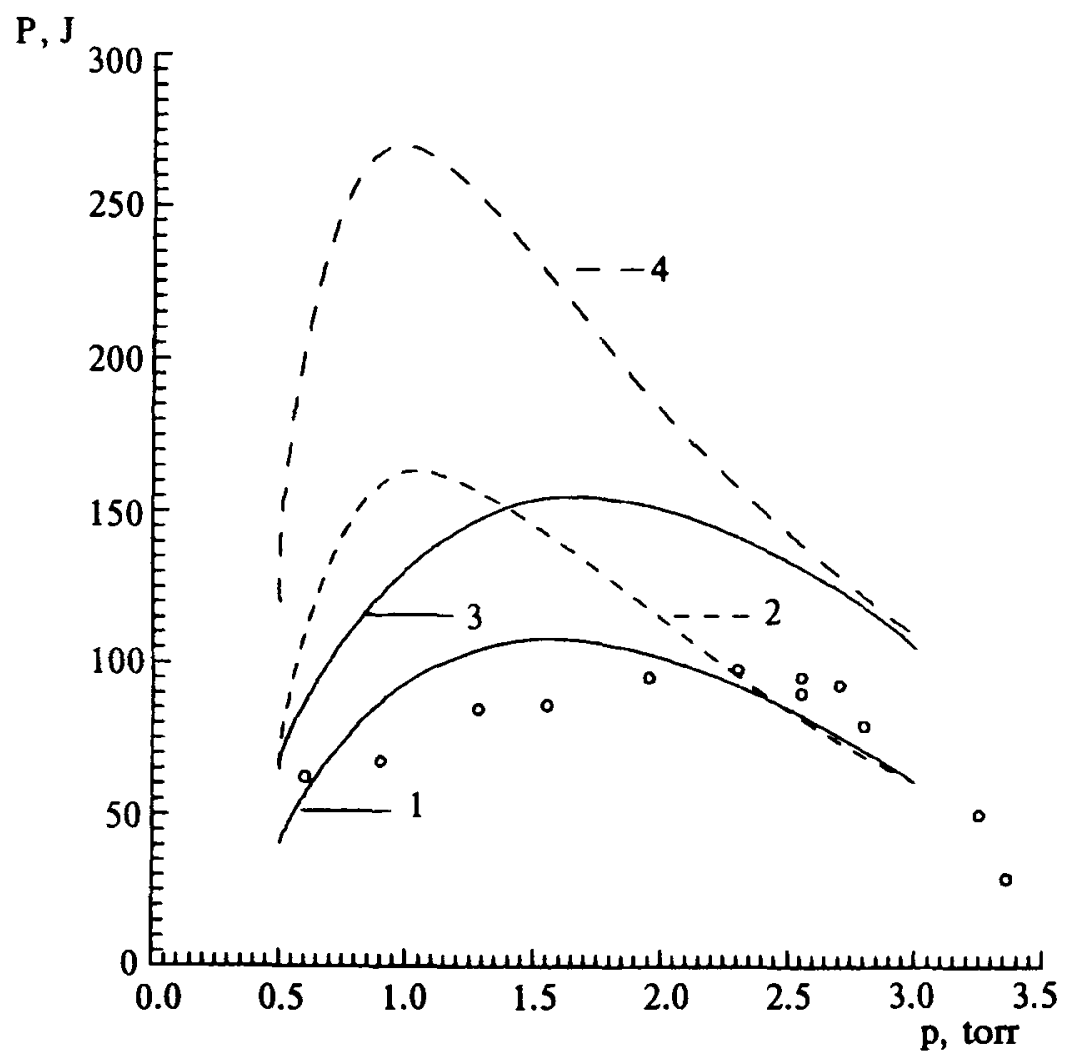

Fig. 7. Dependence of the laser output energy on $\mathrm{XeF}_{2}$ pressure for $\mathrm{N}_{2}$ pressure of 270 torr, Ar pressure of 790 torr, and mirror reflectivities $R_{1}=0.99$ and $R_{2}=0.77: 1$ ) calculation for the conditions of [7],2) the same as 1 but with an increased laser aperture, 3) the same as 1 but $L_{a}=L_{c}=190 \mathrm{~cm}$, and 4) the same as 3 but with an increased laser aperture. Circles o represent the results of experiment [7].

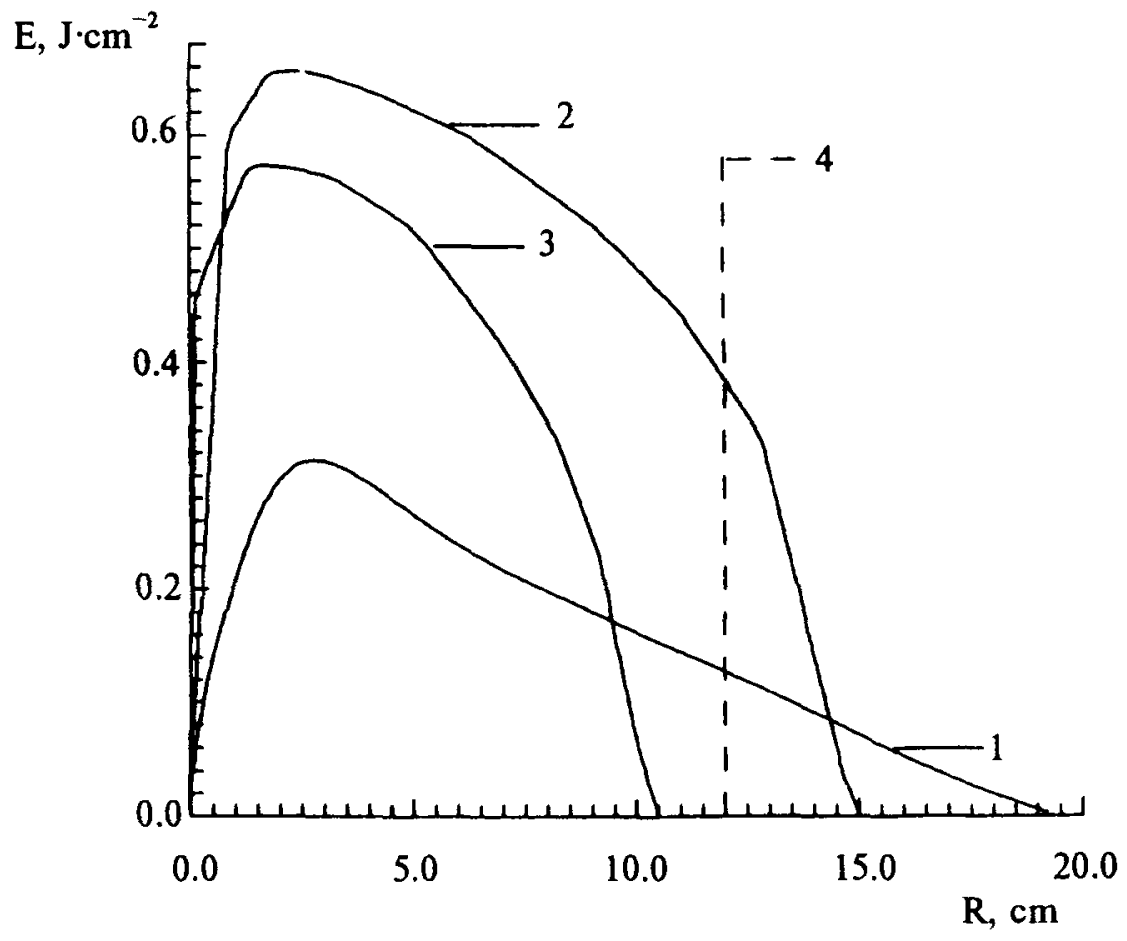

Fig. 8. Laser output energy density versus distance from the discharge under the conditions of experiment [7] and $L_{a}=190 \mathrm{~cm}$ and $L_{c}=280 \mathrm{~cm}$ for different values of $\mathrm{XeF}_{2}$ pressure: 1) $\left.0.5 \mathrm{torr}, 2\right) 1.5 \mathrm{torr}$, and 3) 3.0 torr. Dashed line 4 shows the position of the laser chamber wall. 


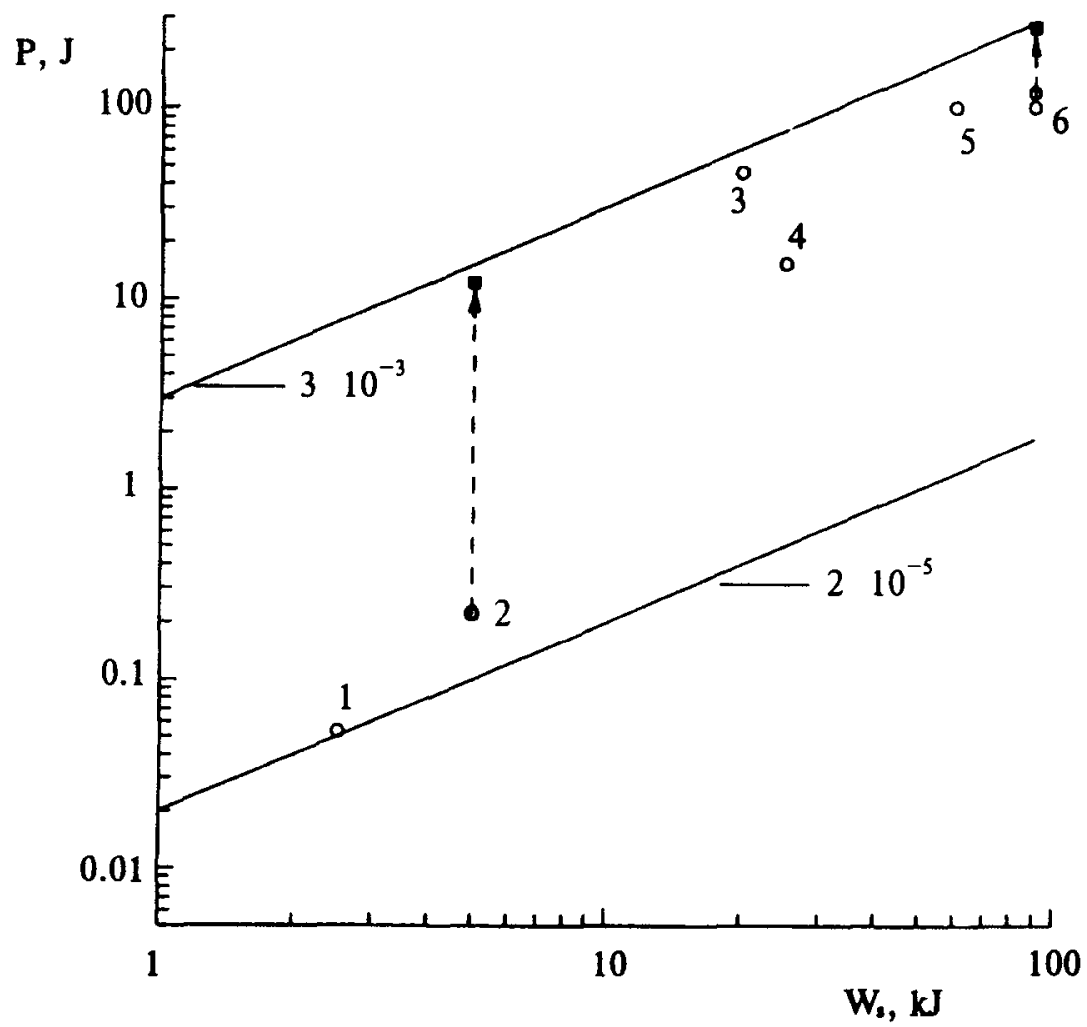

Fig. 9. Output energy versus stored energy for $\mathrm{XeF}(\mathrm{C}-\mathrm{A})$ laser according to different references: 1) [8], 2) [9], 3) [6], 4) [3], 5) [5], and 6) [7]. Dashed arrow shows the possible increase in the output energy calculated in the present paper.

with an increased aperture. It worth noting that all modern photolysis-pumped $\mathrm{XeF}(\mathrm{C}-\mathrm{A})$ lasers operate at $L_{a} / L_{c} \sim 0.5$ and it is important to verify our prediction experimentally because it provides a simple possibility for considerable improvement of laser performance. The output energies of optically pumped $\mathrm{XeF}(\mathrm{C}-\mathrm{A})$ lasers known up to now are plotted in Fig. 9 versus the energy stored in the capacitor. The possible increase in output energy is also shown for the two lasers described above. The solid curves correspond to a constant efficiency. It is seen that under optimum conditions the efficiency of a laser with a small input and that of a larger device are similar.

\section{Conclusion}

The intracavity losses caused by refraction considerably reduce $\mathrm{XeF}(\mathrm{C}-\mathrm{A})$ laser efficiency and limit the upper level of $\mathrm{XeF}_{2}$ pressure to 3-4 torr in the case of a plane-parallel cavity.

The output energy strongly depends on the geometry factor $\mathrm{L}_{a} / \mathrm{L}_{c}$. This makes it possibile to optimize laser construction.

The efficiency of a laser with a small energy input may be close to that of a larger laser under optimum conditions. The maximum value of the technical efficiency is estimated to be $(2.5-3) \cdot 10^{-3}$. 
TABLE 1

\begin{tabular}{|c|c|}
\hline Reaction & Rate constant, Refs. $[4,10]$ \\
\hline $\begin{array}{c}\text { Photoexcitation } \\
\begin{aligned} \mathrm{XeF}_{2}+\mathrm{h} \nu & \sim 158 \mathrm{~nm}) \rightarrow \mathrm{XeF}(\mathrm{B})+\mathrm{F} \\
& \rightarrow \mathrm{XeF}(\mathrm{C})+\mathrm{F} \\
& \rightarrow \mathrm{XeF}(\mathrm{X})+\mathrm{F}\end{aligned}\end{array}$ & $\begin{array}{c}f(\mathrm{~B})=0.85 \\
f(\mathrm{C})=0.035 \\
f(\mathrm{X})=1-f(\mathrm{~B})-f(\mathrm{C})\end{array}$ \\
\hline $\begin{aligned} & \text { Collisional mixing } \\
& \mathrm{XeF}(\mathrm{B})+\mathrm{XeF}_{2} \rightarrow \mathrm{XeF}(\mathrm{C})+\mathrm{XeF}_{2} \\
& \mathrm{XeF}(\mathrm{B})+\mathrm{F}_{2} \rightarrow \mathrm{XeF}(\mathrm{C})+\mathrm{F}_{2} \\
& \mathrm{XeF}(\mathrm{B})+\mathrm{M} \rightarrow \mathrm{XeF}(\mathrm{C})+\mathrm{M}\end{aligned}$ & $\begin{array}{c}\mathrm{k}_{1}=5 \cdot 10^{-11} \\
\mathrm{k}_{2}=2.4 \cdot 10^{-11} \\
\mathrm{k}_{3}=8.6 \cdot 10^{-12}(\mathrm{M}=\mathrm{Ar}) \\
3.4 \cdot 10^{-11}(\mathrm{M}=\mathrm{Kr}) \\
4.4 \cdot 10^{-11}\left(\mathrm{M}=\mathrm{N}_{2}\right)\end{array}$ \\
\hline $\begin{aligned} \mathrm{XeF}(\mathrm{B})+\mathrm{XeF}_{2} & \rightarrow \mathrm{XeF}(\mathrm{X})+\mathrm{XeF}_{2} \\
\mathrm{XeF}(\mathrm{C})+\mathrm{XeF}_{2} & \rightarrow \mathrm{XeF}(\mathrm{X})+\mathrm{XeF}_{2} \\
\mathrm{XeF}(\mathrm{B})+\mathrm{F}_{2} & \rightarrow \mathrm{XeF}(\mathrm{X})+\mathrm{F}_{2} \\
\mathrm{XeF}(\mathrm{C})+\mathrm{F}_{2} & \rightarrow \mathrm{XeF}(\mathrm{X})+\mathrm{F}_{2} \\
\mathrm{XeF}(\mathrm{B})+\mathrm{F} & \rightarrow \mathrm{XeF}(\mathrm{X})+\mathrm{F} \\
\mathrm{XeF}(\mathrm{C})+\mathrm{F} & \rightarrow \mathrm{XeF}(\mathrm{X})+\mathrm{F}\end{aligned}$ & $\begin{array}{c}\mathbf{k}_{4}=5 \cdot 10^{-14}(\mathrm{M}=\mathrm{Ar}) \\
1 \cdot 10^{-13}(\mathrm{M}=\mathrm{Kr}) \\
1 \cdot 10^{-13}\left(\mathrm{M}=\mathrm{N}_{2}\right) \\
\mathrm{k}_{5}=3.5 \cdot 10^{-10} \\
\mathrm{k}_{6}=2 \cdot 10^{-10} \\
\mathrm{k}_{7}=3.8 \cdot 10^{-10} \\
\mathrm{k}_{8}=3.8 \cdot 10^{-10} \\
\mathrm{k}_{9}=1.1 \cdot 10^{-9} \\
\mathrm{k}_{10}=1.1 \cdot 10^{-9}\end{array}$ \\
\hline 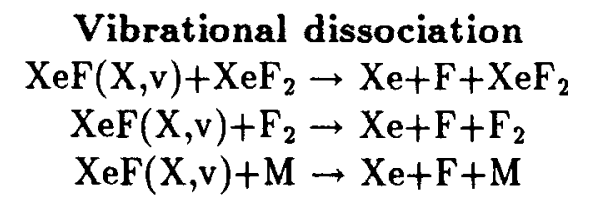 & $\begin{array}{c}\lambda_{1}=1.89 \cdot 10^{-11} \\
\lambda_{2}=9 \cdot 10^{-13} \\
\lambda_{3}=7.4 \cdot 10^{-13}\end{array}$ \\
\hline $\begin{array}{ll} & \text { Emission } \\
\mathrm{XeF}(\mathrm{B}) \rightarrow \mathrm{XeF}(\mathrm{X})+\mathrm{h} \nu(351 \mathrm{~nm}) \\
\mathrm{XeF}(\mathrm{C}) \rightarrow \mathrm{XeF}(\mathrm{A})+\mathrm{h} \nu(480 \mathrm{~nm})\end{array}$ & $\begin{aligned} \mathrm{A}_{\mathrm{B}}=7.7 \cdot 10^{7}, \sigma_{\mathrm{BX}} & =1.2 \cdot 10^{-15} \\
\mathrm{~A}_{\mathrm{C}}=1 \cdot 10^{7}, \sigma_{\mathrm{CA}} & =9 \cdot 10^{-18}\end{aligned}$ \\
\hline $\begin{array}{c}\text { Dimerization } \\
\mathrm{F}+\mathrm{F}+\mathrm{XeF}_{2} \rightarrow \mathrm{F}_{2}+\mathrm{XeF}_{2} \\
\mathrm{~F}+\mathrm{F}+\mathrm{F}_{2} \rightarrow 2 \mathrm{~F}_{2} \\
\mathrm{~F}+\mathrm{F}+\mathrm{M} \rightarrow \mathrm{F}_{2}+\mathrm{M}\end{array}$ & $\begin{aligned} z_{1} & =2.93 \cdot 10^{-31} \\
z_{2} & =1.96 \cdot 10^{-31} \\
z_{3} & =8.2 \cdot 10^{-31}\end{aligned}$ \\
\hline
\end{tabular}




\section{Appendix}

\section{$\mathrm{XeF}(\mathrm{C}-\mathrm{A})$ Laser Kinetics}

In Table 1 the employed units are: $f(\mathrm{~B}, \mathrm{C}, \mathrm{X})-$ dimensionless, $k\left[\mathrm{~cm}^{3} / \mathrm{s}\right], \lambda\left[\mathrm{cm}^{3} / \mathrm{s}\right], z\left[\mathrm{~cm}^{6} / \mathrm{s}\right], T[\mathrm{~K}]$, $A\left[\mathrm{~s}^{-1}\right]$, and $\sigma\left[\mathrm{cm}^{2}\right]$. The A state is assumed to disintegrate rapidly, i.e., $\mathrm{XeF}(\mathrm{A}) \mathrm{L} \rightarrow \mathrm{Xe}+\mathrm{F}$.

The system of equations describing $\mathrm{XeF}(\mathrm{C}-\mathrm{A})$ laser kinetics has the form

$$
\begin{aligned}
\frac{d[\mathrm{XeF}(\mathrm{B})]}{d t} & =-f(\mathrm{~B}) \frac{d\left[\mathrm{XeF}_{2}\right]}{d t}-[\mathrm{XeF}(\mathrm{B})] \nu_{1} \\
\frac{d[\mathrm{XeF}(\mathrm{C})]}{d t} & =-f(\mathrm{C}) \frac{d\left[\mathrm{XeF}_{2}\right]}{d t}+[\mathrm{XeF}(\mathrm{B})] \nu_{2}-[\mathrm{XeF}(\mathrm{C})] \eta_{1}-c \sigma_{\mathrm{CA}} \Phi \\
\frac{d[\mathrm{XeF}(\mathrm{X})]}{d t} & =-f(\mathrm{X}) \frac{d\left[\mathrm{XeF}_{2}\right]}{d t}+[\mathrm{XeF}(\mathrm{B})] \nu_{3}+[\mathrm{XeF}(\mathrm{C})] \eta_{2}-[\mathrm{XeF}(\mathrm{X})] \omega_{1} \\
\frac{d[\mathrm{~F}]}{d t} & =-\frac{d\left[\mathrm{XeF}_{2}\right]}{d t}+[\mathrm{XeF}(\mathrm{X})] \omega_{1}-[\mathrm{F}]^{2} \omega_{2}+\left(A_{c}+c \sigma_{\mathrm{CA}} \Phi\right)[\mathrm{XeF}(\mathrm{C})] \\
\frac{d\left[\mathrm{XeF}_{2}\right]}{d t} & =[\mathrm{F}]^{2} \omega_{2},
\end{aligned}
$$

where $\left[\mathrm{XeF}_{2}\right],[\mathrm{XeF}(\mathrm{B})],[\mathrm{XeF}(\mathrm{C})]$, etc. are the densities of the species in $\mathrm{cm}^{-3}$ and

$$
\begin{aligned}
\nu_{1} & =\left(k_{1}+k_{5}\right)\left[\mathrm{XeF}_{2}\right]+\left(k_{2}+k_{7}\right)\left[\mathrm{F}_{2}\right]+k_{9}[\mathrm{~F}]+\left(k_{3}+k_{4}\right) M+A_{b} \\
\nu_{2} & =k_{1}\left[\mathrm{XeF}_{2}\right]+k_{2}\left[\mathrm{~F}_{2}\right]+k_{3} M \\
\nu_{3} & =k_{5}\left[\mathrm{XeF}_{2}\right]+k_{7}\left[\mathrm{~F}_{2}\right]+k_{9}[\mathrm{~F}]+A_{b}+k_{4} M \\
\eta_{1} & =k_{6}\left[\mathrm{XeF}_{2}\right]+k_{8}\left[\mathrm{~F}_{2}\right]+k_{10}[\mathrm{~F}]+A_{c} \\
\eta_{2} & =k_{6}\left[\mathrm{XeF}_{2}\right]+k_{8}\left[\mathrm{~F}_{2}\right]+k_{10}[\mathrm{~F}]+k_{4} M \\
\omega_{1} & =\lambda_{1}\left[\mathrm{XeF}_{2}\right]+\lambda_{2}\left[\mathrm{~F}_{2}\right]+\lambda_{3} M \\
\omega & =z_{1}\left[\mathrm{XeF}_{2}\right]+z_{2}\left[\mathrm{~F}_{2}\right]+z_{3} M
\end{aligned}
$$

\section{References}

1. W. K. Bischel, H. Nakano, D. J. Eckstrom, et al., Appl. Phys. Lett., 34, 565 (1979); Postdeadline Paper presented at the Intern. Conference on Lasers, Orlando, Florida (1978).

2. N. G. Basov, V. S. Zuev, A. V. Kanaev, et al., Sov. J. Quantum Electron., 9, 629 (1979).

3. V. S. Zuev, L. D. Mikheev, and D. B. Stavrovskii, Sov. J. Quantum Electron., 14, 1174 (1984).

4. L. D. Mikheev, D. B. Stavrovskii, and V. S. Zuev, J. Russ. Laser Res., 16, 427 (1995).

5. V. S. Zuev, G. N. Kashikov, N. P. Kozlov, et al., Sov. J. Quantum Electron., 16, 1665 (1986).

6. V. S. Zuev, G. N. Kashnikov, V. V. Kirilenko, et al., Sov. J. Quantum Electron., 19, 748 (1989).

7. V. S. Zuev, G. N. Kashnikov, and S. B. Mamaev, "Investigations of XeF laser optically pumped by radiation of surface discharges," Preprint of the Lebedev Physical Institute, No. 23, Moscow (1992).

8. B. A. Knecht, R. D. Fraser, D. J. Wheeler, et al., Opt. Lett., 20, 1011 (1995).

9. S. V. Mitko, F. A. van Goor, W. J. Witteman, and V. N. Ochkin, "Optical pumping and ferrite flash discharges," in: V. N. Ochkin and W. J. Witteman (eds.), Proceedings of the NATO Advanced Reseach Workshop ARW 950443 on Gas Lasers-Recent Developments and Future Prospects, Moscow, July 2-5, 1995, Kluwer, Dordrecht (1996), p. 185. 
10. R. E. Beverly III, Appl. Phys. B, 56, 147 (1993).

11. N. K. Bibinov, L. P. Vinogradov, L. D. Mikheev, and D. B. Stavrovskii, Sov. J. Quantum Electron., 11, 1178 (1981).

12. E. L. Latush and G. D. Chebotarev, Kvantov. Élektron., 12, 1480 (1985).

13. W. J. Witteman, The $\mathrm{CO}_{2}$ Laser, Springer, Berlin (1987).

14. D. B. Stavrovskii, "Photodissociation XeF-Laser," Ph.D. Thesis, Lebedev Physical Institute, Moscow (1987).

15. V. S. Zuev, A. V. Kanaev, and L. D. Mikheev, Sov. J. Quantum Electron., 13, 1232 (1983).

16. I. K. Kikoin, Tables of Physical Constants. Handbook [in Russian], Atomizdat, Moscow (1976).

17. E. P. Orlov, "Theory of the Lasers with Refractive Losses," Ph.D. Thesis, Lebedev Physical Institute, Moscow (1985). 